\title{
Asymptotic properties of Urysohn type generalized sampling operators
}

\section{Karsli H.}

\begin{abstract}
The concern of this study is to continue the investigation of convergence properties of Urysohn type generalized sampling operators, which are defined by the author in [Dolomites Res. Notes Approx. 2021, 14 (2), 58-67]. In details, the paper centers around to investigation of the asymptotic properties together with some Voronovskaya-type theorems for the linear and nonlinear counterpart of Urysohn type generalized sampling operators.

Key words and phrases: generalized sampling operator, linear and nonlinear Urysohn type generalized sampling operator, asymptotic expansion, Voronovskaya-type theorem.
\end{abstract}

Bolu Abant Izzet Baysal University, Bolu, Turkey

E-mail: karsli_h@ibu.edu.tr

\section{Introduction}

The theory of the generalized sampling series, introduced and developed at RWTH Aachen by Paul Leo Butzer, is a powerful tool for investigating and proving the approximation problem on $\mathbb{R}$. Indeed, from mathematical point of view, generalized sampling operators of functions $f$ defined on the real axis, which are not necessarily band-limited, were extensively and systematicaly studied by P.L. Butzer and his collaborators in RWTH Aachen since 1977 (see, e.g., $[11-15,32])$.

Let $f$ be a bounded function defined on $\mathbb{R}$, then the generalized sampling series $\left(S_{n} f\right)$ is given by

$$
\left(S_{n} f\right)(t):=\sum_{k=-\infty}^{\infty} f\left(\frac{k}{n}\right) \varphi(n t-k), \quad t \in \mathbb{R}, n \in \mathbb{N},
$$

where $\varphi: \mathbb{R} \rightarrow \mathbb{R}$ is the kernel function satisfying

$$
\varphi \in L^{1}, \quad \sum_{k=-\infty}^{\infty} \varphi(u-k)=1 \quad \text { for every } u \in \mathbb{R} \text {, }
$$

and

$$
A_{\varphi}:=\sup _{u \in \mathbb{R}} \sum_{k=-\infty}^{\infty}|\varphi(u-k)|<\infty,
$$

where the convergence of the series (3) is uniform on each compact subintervals of $\mathbb{R}$.

These series allow us to reconstruct a given signal (function) $f$ defined on $\mathbb{R}$ by its rational sampling values, which are of the form $f(k / n), k \in \mathbb{Z}, n \in \mathbb{N}$. 
Especially in the last decade, these operators and some of their modifications have been of great importance in the development of mathematical models for signal and image recovering, as studied by research group (RITA Network) from Perugia led by Carlo Bardaro and Gianluca Vinti (see, e.g., [1-10,16-19,31]).

In [28], the author introduced Urysohn type generalized sampling operators by replacing the rational sampling values of the function $f$ with its Urysohn type operator values. By these new operators the convergence problems generalized and extended to the functionals and operators. Once a detailed model of the aforementioned operators has been constructed in [28], the next step is to investigate their approximation and asymptotic properties. As a continuation of the study of the author [28], the present work highlights the importance of Urysohn type generalized sampling operators and in modelling the asymptotic approximation properties of functions $f$ defined on the whole real line.

In particular, the goal of this study is to obtain asymptotic expansion and some Voronovskaya type problems for newly defined Urysohn type generalized sampling operators. We also define and investigate some asymptotic properties of its nonlinear counterpart, called nonlinear Urysohn type generalized sampling operators.

\section{Preliminaries}

In this section, we shall introduce some notation and background material used throughout this work.

As in the papers $[6,18,28]$, throughout this work, we assume that the first two central moments of the generalized sampling operators (1) satisfy

$$
\begin{aligned}
& m_{1}(\varphi):=\sum_{k=-\infty}^{\infty} \varphi(u-k)(u-k)=0 \\
& m_{2}(\varphi):=\sum_{k=-\infty}^{\infty} \varphi(u-k)(u-k)^{2}=C
\end{aligned}
$$

for every $u \in \mathbb{R}$ and for a given constant $C \in \mathbb{R}$.

We also assume that the discrete absolute moment of order $\beta$ are finite, i.e.

$$
M_{\beta}(\varphi):=\sup _{u \in \mathbb{R}} \sum_{k=-\infty}^{\infty}|\varphi(u-k)||u-k|^{\beta}<\infty
$$

for every $u \in \mathbb{R}$ and for some $\beta>0$. The formula for $M_{\beta}(\varphi)$ in case $\beta=0$ is exactly $A_{\varphi}$.

To define an Urysohn type operator and obtain some positive answers to the approximation problems, H. Karsli [28] considered the following Urysohn integral operators discussed by P. Urysohn in [33]:

$$
F(t, x(\cdot))=\int_{0}^{1} f(t, s, x(s)) d s, \quad t \in[0,1], \quad 0 \leq x(\cdot) \leq 1,
$$

with unknown kernel $f$, whose properties and values depend on the function $x(\cdot)$ (see, e.g., $[20,23-28,30])$.

For a constant function $x(\cdot)=a$, we set $F a(t)=F(a)$. 
In view of the relations between Dirac and Heaviside unit step functions, we assume that the following continuous interpolation condition holds

$$
F x(t):=F\left(t, x_{i}(\cdot, s)\right)=\int_{0}^{1} f\left(t, z, x_{i}(z, s)\right) d z, \quad t \in[0,1]
$$

where $-\infty<x_{i}(z, s)<\infty$ defined as $x_{i}(z, s)=(i / n) H(z-s), s \in[0,1]$, and $i \in \mathbb{Z}$.

Obviously

$$
\frac{\partial F\left(t, x_{i}(\cdot, s)\right)}{\partial s}=\frac{\partial F(t,(i / n) H(\cdot-s))}{\partial s}=f(t, s, 0)-f\left(t, s, \frac{i}{n}\right)
$$

holds true, where $x_{i}(\cdot, s)=(i / n) H(\cdot-s), s \in[0,1]$, and $i \in \mathbb{Z}$.

In [28], H. Karsli constructed a new type of generalized sampling operator by means of the Urysohn interpolation conditions given in (7) with unknown function $f:[0,1]^{2} \times \mathbb{R} \rightarrow \mathbb{R}$, which he plan to use for the solution of the convergence problem to the Urysohn operator.

Now we are able to recall the definition of the Urysohn type generalized sampling operators constructed by the author.

Definition 1 ([28]). Let $F$ be the Urysohn integral operator of $f$. Then the Urysohn type generalized sampling operator is defined as

$$
\left(U S_{n} F\right) x(t):=\left(U S_{n} F\right)(t ; x(\cdot, s))=\int_{0}^{1}\left[\sum_{k=-\infty}^{\infty} f\left(t, s, \frac{k}{n}\right) \varphi_{k, n}(x(s))\right] d s,
$$

where $\varphi_{k, n}(x(s))=\varphi(n x(s)-k)$ is an arbitrary kernel function satisfying (2) and (3).

Here, Dom $(U S F)=\bigcap_{n \in \mathbb{N}} \operatorname{Dom}\left(U S_{n} F\right)$, where Dom $\left(U S_{n} F\right)$ is the set of all bounded functions $f:[0,1]^{2} \times \mathbb{R} \rightarrow \mathbb{R}$ for which the operator is well defined.

Remark 1. By (6) and (8), $\left(U S_{n} F\right)$ can be written as

$$
\left(U S_{n} F\right) x(t)=F(0)-\int_{0}^{1}\left[\sum_{k=-\infty}^{\infty} \frac{\partial F(t,(k / n) H(t-s))}{\partial s} \varphi_{k, n}(x(s))\right] d s .
$$

\section{Asymptotic expansion and Voronovskaya-type theorems}

This section deals with an asymptotic formula and some Voronovskaya type theorems for the Urysohn type generalized sampling operators.

Theorem 1. Let $F$ be the Urysohn integral operator of the bounded function $f:[0,1]^{2} \times \mathbb{R} \rightarrow \mathbb{R}$. Moreover, for a certain $r \in \mathbb{N}$, we also assume that $\partial^{r} f(t, s, x(s)) / \partial x^{r}$ exists at a fixed point $x(s)$. Then the following asymptotic formula

$$
\left(U S_{n} F\right) x(t)=F x(t)+\sum_{i=1}^{r} \frac{m_{i}\left(\varphi_{k, n}(x(s))\right)}{i ! n^{i}} \frac{\partial^{i}}{\partial x^{i}} f(t, s, x(s))+o\left(n^{-r}\right) \quad \text { as } n \rightarrow \infty
$$

holds, where $m_{i}$ is the ith order moment. 
Proof. Since $\partial^{r} f(t, s, x(s)) / \partial x^{r}$ exists at a point $x(s)$, then there exists a bounded function $h$ such that $\lim _{y \rightarrow 0} h(t, s, y)=0$. By the local Taylor's formula we have

$$
f\left(t, s, \frac{k}{n}\right)=\sum_{i=0}^{r} \frac{(k / n-x(s))^{i}}{i ! n^{i}} \frac{\partial^{i}}{\partial x^{i}} f(t, s, x(s))+h\left(t, s, \frac{k}{n}-x(s)\right)\left(\frac{k}{n}-x(s)\right)^{r} .
$$

In view of (9) and (10), we can write

$$
\begin{aligned}
\left(U S_{n} F\right) x(t)= & \int_{0}^{1}\left[\sum_{k=-\infty}^{\infty} f\left(t, s, \frac{k}{n}\right) \varphi_{k, n}(x(s))\right] d s \\
= & \int_{0}^{1}\left[\sum _ { k = - \infty } ^ { \infty } \left(\sum_{i=0}^{r} \frac{(k / n-x(s))^{i}}{i ! n^{i}} \frac{\partial^{i}}{\partial x^{i}} f(t, s, x(s))\right.\right. \\
& \left.\left.+h\left(t, s, \frac{k}{n}-x(s)\right)\left(\frac{k}{n}-x(s)\right)^{r}\right) \varphi_{k, n}(x(s))\right] d s \\
= & \int_{0}^{1}\left[\sum_{k=-\infty}^{\infty}\left(\sum_{i=0}^{r} \frac{(k / n-x(s))^{i}}{i ! n^{i}} \frac{\partial^{i}}{\partial x^{i}} f(t, s, x(s))\right) \varphi_{k, n}(x(s))\right] d s \\
& +\int_{0}^{1}\left[\sum_{k=-\infty}^{\infty}\left(h\left(t, s, \frac{k}{n}-x(s)\right)\left(\frac{k}{n}-x(s)\right)^{r}\right) \varphi_{k, n}(x(s))\right] d s=: I_{1}+R .
\end{aligned}
$$

Let us analyze the terms $I_{1}$ and $R$, respectively. Let us consider the term $I_{1}$.

$$
\begin{aligned}
I_{1}= & \int_{0}^{1}\left[\sum_{k=-\infty}^{\infty}\left(\sum_{i=0}^{r} \frac{(k / n-x(s))^{i}}{i ! n^{i}} \frac{\partial^{i}}{\partial x^{i}} f(t, s, x(s))\right) \varphi_{k, n}(x(s))\right] d s \\
= & \int_{0}^{1}\left[\sum_{k=-\infty}^{\infty} f(t, s, x(s)) \varphi_{k, n}(x(s))\right] d s \\
& +\int_{0}^{1}\left[\sum_{k=-\infty}^{\infty}\left(\sum_{i=1}^{r} \frac{(k / n-x(s))^{i}}{i ! n^{i}} \frac{\partial^{i}}{\partial x^{i}} f(t, s, x(s))\right) \varphi_{k, n}(x(s))\right] d s \\
= & F x(t)+\sum_{i=1}^{r} \frac{1}{i ! n^{i}}\left(\frac{\partial^{i}}{\partial x^{i}} f(t, s, x(s))\right) \int_{0}^{1}\left[\sum_{k=-\infty}^{\infty}\left(\frac{k}{n}-x(s)\right)^{i} \varphi_{k, n}(x(s))\right] d s \\
= & F x(t)+\sum_{i=1}^{r} \frac{m_{i}\left(\varphi_{k, n}(x(s))\right)}{i ! n^{i}} \frac{\partial^{i}}{\partial x^{i}} f(t, s, x(s)) .
\end{aligned}
$$

Now, we evaluate the remainder term $R$.

Let $\varepsilon>0$ be fixed. Since $h(t, s, y)$ is a bounded function such that $\lim _{y \rightarrow 0} h(t, s, y)=0$, there exists $\delta>0$ such that $|h(y)| \leq \varepsilon$ for every $|y| \leq \delta$. In view of the assumption (5), clearly we have

$$
R=\int_{0}^{1}\left[\left(\sum_{|k / n-x(s)| \geq \delta}+\sum_{|k / n-x(s)|<\delta}\right) h\left(t, s, \frac{k}{n}-x(s)\right)\left(\frac{k}{n}-x(s)\right)^{r} \varphi_{k, n}(x(s))\right] d s=o\left(n^{-r}\right) .
$$

As a consequence of Theorem 1 we can establish the following first and second order Voronovskaya type theorems, respectively. 
Theorem 2. Let $f$ be a bounded function such that $\partial f(t, s, x(s)) / \partial x$ exists at a point $x(s)$ and let $F$ be its corresponding Urysohn integral operator. Then we have

$$
\lim _{n \rightarrow \infty} n\left[\left(U S_{n} F\right) x(t)-F x(t)\right]=m_{1}(x(s)) \frac{\partial}{\partial x} f(t, s, x(s)) .
$$

Proof. Applying the asymptotic formula of Theorem 1 with $r=1$, using (10) and assumption (4), we can write

$$
\left(U S_{n} F\right) x(t)=F x(t)+\frac{m_{1}\left(\varphi_{k, n}(x(s))\right)}{n} \frac{\partial}{\partial x} f(t, s, x(s))+o\left(n^{-1}\right) \quad \text { as } n \rightarrow \infty .
$$

Then the proof follows by passing to the limit for $n \rightarrow \infty$.

Theorem 3. Let $f$ be a bounded function such that $\partial^{2} f(t, s, x(s)) / \partial x^{2}$ exists at a point $x(s)$ and let $F$ be its corresponding Urysohn integral operator. Then we have

$$
\lim _{n \rightarrow \infty} n^{2}\left[\left(U S_{n} F\right) x(t)-F x(t)\right]=m_{1}(x(s)) \frac{\partial}{\partial x} f(t, s, x(s))+\frac{m_{2}(x(s))}{2} \frac{\partial^{2}}{\partial x^{2}} f(t, s, x(s)) .
$$

Proof. As in the proof of Theorem 2, applying the asymptotic formula of Theorem 1 with $r=2$, using (10) and assumption (4), we can write

$$
\left(U S_{n} F\right) x(t)=F x(t)+\sum_{i=1}^{2} \frac{m_{i}\left(\varphi_{k, n}(x(s))\right)}{i ! n^{i}} \frac{\partial^{i}}{\partial x^{i}} f(t, s, x(s))+o\left(n^{-2}\right) \quad \text { as } n \rightarrow \infty .
$$

Then the proof follows by passing to the limit for $n \rightarrow \infty$.

The above theorems show that the order of pointwise approximation is at least of order $O\left(n^{-1}\right)$ and $O\left(n^{-2}\right)$ as $n \rightarrow+\infty$, respectively.

\section{Nonlinear counterpart and Voronovskaya-type theorems}

We now introduce some notations and structural hypotheses for nonlinear approximation, which will be fundamental in proving our theorems.

Let $X$ be the set of all bounded Lebesgue measurable functions $f:[0,1]^{3} \rightarrow \mathbb{R}_{0}^{+}=[0, \infty)$. Let $\Psi$ be the set of all continuous, concave and non-decreasing functions $\varphi: \mathbb{R}_{0}^{+} \rightarrow \mathbb{R}_{0}^{+}$with $\varphi(0)=0, \varphi(u)>0$ for all $u>0$ and $\lim _{u \rightarrow+\infty} \varphi(u)=+\infty$ in the usual sense. Such a function is called a $\varphi$-function (see [6-10]).

We now introduce a sequence of functions. Let $\left\{P_{k, n}\right\}_{n \in \mathbb{N}}$ be a sequence of functions $P_{k, n}:[0,1] \times \mathbb{R} \rightarrow \mathbb{R}$ defined by

$$
P_{k, n}(t, u)=\varphi_{k, n}(t) H_{n}(u)
$$

for every $t \in[0,1], u \in \mathbb{R}$, where $H_{n}: \mathbb{R} \rightarrow \mathbb{R}$ is such that $H_{n}(0)=0$ and $\varphi_{k, n}(t)$ again $\varphi_{k, n}(x(s))=\varphi(n x(s)-k)$ is an arbitrary kernel function satisfies (2) and (3).

Throughout the paper we assume that $\mu: \mathbb{N} \rightarrow \mathbb{R}^{+}$is an increasing and continuous function such that $\lim _{n \rightarrow \infty} \mu(n)=\infty$.

Assume that the function $H_{n}$ satisfies following conditions:

a) $H_{n}: \mathbb{R} \rightarrow \mathbb{R}$ is such that $\left|H_{n}(u)-H_{n}(v)\right| \leq \psi(|u-v|)$ holds for every $u, v \in \mathbb{R}$, for every $n \in \mathbb{N}$, that is, $H_{n}$ satisfies a $(\Psi)$ Lipschitz condition; 
b) denoting by $r_{n}(u):=H_{n}(u)-u, u \in \mathbb{R}$ and $n \in \mathbb{N}$, such that

$$
\sup _{u}\left|r_{n}(u)\right|=\sup _{u}\left|H_{n}(u)-u\right| \leq \frac{1}{\mu(n)}
$$

uniformly with respect to $u$ or $n$ sufficiently large.

Owing to the above definitions, we introduce nonlinear counterpart of the Urysohn type generalized sampling operators as follows.

Definition 2. Let $F$ be the Urysohn integral operator of $f$. In view of (7) and (11), we define the following Urysohn type nonlinear generalized sampling operators as

$$
\left(N U S_{n} F\right) x(t)=\int_{0}^{1}\left[\sum_{k=-\infty}^{\infty} P_{k, n}\left(x(s), f\left(t, s, \frac{k}{n}\right)\right)\right] d s,
$$

where $n$ is a non-negative integer, $P_{k, n}$ satisfy some suitable assumptions.

Definition 3. We will say that the sequence $\left(P_{n}\right)_{n \in \mathbb{N}}$ is $(\psi-\alpha)$-singular if the following assumptions are satisfied:

(P.1) for every $x \in I$ and $\delta>0$ there holds

$$
\psi\left(\sum_{|k / n-x| \geq \delta}\left|\frac{k}{n}-x\right| \varphi_{k, n}(x)\right)=o\left(n^{-\alpha}\right) \quad \text { as } n \rightarrow \infty ;
$$

(P.2) for every $u \in \mathbb{R}$ and for every $x \in I$ we have

$$
\lim _{n \rightarrow \infty} n^{\alpha}\left[\sum_{k=0}^{n} P_{n, k}(x, u)-u\right]=0 .
$$

Note that since the theory of approximation via nonlinear operators is quite different from its linear counterpart, in same cases we can obtain only some estimates related with the convergence problems (see [10,21,22]). Actually, in some cases, it is not possible to obtain exact estimates for nonlinear operators, because of the nonlinearity of their kernel functions (see $[6,7,29])$.

The first approximation result on the nonlinear counterpart of the Urysohn operators is the following assertion.

Theorem 4. Let $f$ be a bounded function such that $\partial f(t, s, x(s)) / \partial x$ exists at a point $x(s)$ and let $F$ be its corresponding Urysohn integral operator. Let us also assume that the sequence $\left(P_{n, k}\right)_{n \in \mathbb{N}}$ is $(\psi-1)$-singular with

$$
\limsup _{n \rightarrow \infty} n \psi\left(M_{1}\left(\varphi_{k, n}(x(s))\right)\right)=l_{1}(x(s)) \in \mathbb{R},
$$

where $M_{1}(\varphi)$ is the first order absolute moment of generalized sampling operators. Then,

$$
\limsup _{n \rightarrow \infty} n\left|\left(N U S_{n} F\right) x(t)-F x(t)\right| \leq C l_{1}(x(s))
$$

holds true, where $C>0$ is a sufficiently large constant. 
Proof. Since $f$ is differentiable at the point $x(s)$ then there exists a bounded function $h$ such that $\lim _{y \rightarrow 0} h(t, s, y)=0$. By Taylor's formula we have

$$
f\left(t, s, \frac{k}{n}\right)=f(t, s, x(s))+\left(\frac{k}{n}-x(s)\right) \frac{\partial}{\partial x} f(t, s, x(s))+\left(\frac{k}{n}-x(s)\right) h\left(t, s, \frac{k}{n}-x(s)\right) .
$$

In view of (11), (12) and conditions $a)-b)$, we can write

$$
\left(N U s_{n} F\right) x(t)=\int_{0}^{1}\left[\sum_{k=-\infty}^{\infty} P_{k, n}\left(x(s), f\left(t, s, \frac{k}{n}\right)\right)\right] d s=\int_{0}^{1} \sum_{k=-\infty}^{\infty} \varphi_{k, n}(x(s)) H_{n}\left(f\left(t, s, \frac{k}{n}\right)\right) d s
$$

and hence

$$
\begin{aligned}
n \mid\left(N U S_{n} F\right) x(t)- & F x(t)|=n| \int_{0}^{1}\left[\sum_{k=-\infty}^{\infty} P_{k, n}\left(x(s), f\left(t, s, \frac{k}{n}\right)\right)\right] d s-F x(t) \mid \\
= & n\left|\int_{0}^{1}\left[\sum_{k=-\infty}^{\infty} P_{k, n}\left(x(s), f\left(t, s, \frac{k}{n}\right)\right)\right] d s-\int_{0}^{1}\left[\sum_{k=-\infty}^{\infty} P_{k, n}(x(s), f(t, s, x(s)))\right] d s\right| \\
& +n\left|\int_{0}^{1}\left[\sum_{k=-\infty}^{\infty} P_{k, n}(x(s), f(t, s, x(s)))\right] d s-F x(t)\right| \\
= & n\left|\int_{0}^{1} \sum_{k=-\infty}^{\infty} \varphi_{k, n}(x(s))\left[H_{n}\left(f\left(t, s, \frac{k}{n}\right)\right)-H_{n}(f(t, s, x(s)))\right] d s\right| \\
& +n\left|\int_{0}^{1}\left[\sum_{k=-\infty}^{\infty} P_{k, n}(x(s), f(t, s, x(s)))-f(t, s, x(s))\right] d s\right|=: I_{1}+I_{2} .
\end{aligned}
$$

By assumption (P.2), $I_{2}$ tends to zero. We can estimate the first term in the following way. Let $C>0$ be a constant such that $|\partial f(t, s, x(s)) / \partial x|+\left|h\left(t, s, \frac{k}{n}-x(s)\right)\right| \leq C$. Using sub-additivity of the function $\psi(x), x \geq 0$, we have

$$
\begin{aligned}
I_{1} & =n \sum_{k=0}^{n} \psi\left(\left|\left(\frac{k}{n}-x(s)\right) \frac{\partial}{\partial x} f(t, s, x(s))+\left(\frac{k}{n}-x(s)\right) h\left(t, s, \frac{k}{n}-x(s)\right)\right|\right) \varphi_{k, n}(x(s)) \\
& \leq n\left\{\sum_{k=0}^{n} \psi\left(C\left|\left(\frac{k}{n}-x(s)\right)\right|\right) \varphi_{k, n}(x(s))\right\} \leq n C\left\{\sum_{k=0}^{n} \psi\left(\left|\left(\frac{k}{n}-x(s)\right)\right|\right) \varphi_{k, n}(x(s))\right\} .
\end{aligned}
$$

In virtue of Jensen's inequality, we can write

$$
I_{1}(x) \leq n C \psi\left(\sum_{k=0}^{n}\left|\left(\frac{k}{n}-x(s)\right)\right| \varphi_{k, n}(x(s))\right)=n C \psi\left(M_{1}\left(\varphi_{k, n} x(s)\right)\right) .
$$

In view of (13), one has

$$
\limsup _{n \rightarrow \infty} n\left|\left(N U S_{n} F\right)(x)-f(x)\right| \leq C l_{1}(x(s)) .
$$

This completes the proof.

Corollary 1. Let $f$ be a bounded function such that $\partial f(t, s, x(s)) / \partial x$ exists at a point. Let us assume that the sequence $\left(P_{n, k}\right)_{n \in \mathbb{N}}$ is $(\psi-1)$-singular satisfying (13) and let $\psi(x(s))=x^{\gamma}(s)$, where $0<\gamma \leq 1$. Then

$$
\limsup _{n \rightarrow \infty} n\left|\left(N U S_{n} F\right) x(t)-F x(t)\right| \leq l_{1}(x(s))\left|\frac{\partial}{\partial x} f(t, s, x(s))\right|^{\gamma}
$$

holds true. 
Theorem 5. Let $f$ be a bounded function such that $\partial^{2} f(t, s, x(s)) / \partial x^{2}$ exists at a point $x(s)$. Let us assume that the sequence $\left(P_{n, k}\right)_{n \in \mathbb{N}}$ is $(\psi-\alpha)$-singular and

$$
\limsup _{n \rightarrow \infty} n^{\alpha} \psi\left(M_{i}\left(\varphi_{k, n} x(s)\right)\right)=l_{i}(x(s)) \in \mathbb{R} \quad \text { for } i=1,2,
$$

where $M_{1}$ and $M_{2}$ are the first and second order absolute moments of generalized sampling operators, respectively. Then,

$$
\begin{aligned}
\limsup _{n \rightarrow \infty} n^{\alpha}\left|\left(\operatorname{NUS}_{n} F\right) x(t)-F x(t)\right| & \leq \psi\left(\left|\frac{\partial}{\partial x} f(t, s, x(s))\right|\right) l_{1}(x(s)) \\
& +\psi\left(\frac{1}{2}\left|\frac{\partial^{2}}{\partial x^{2}} f(t, s, x(s))\right|\right) l_{2}(x(s))
\end{aligned}
$$

holds true.

Proof. Since $f$ is differentiable at the point $x(s)$, using a local Taylor's formula for the function $f$, we obtain that there exists a bounded function $h$ such that $\lim _{y \rightarrow 0} h(t, s, y)=0$ and

$$
\begin{aligned}
f\left(t, s, \frac{k}{n}\right)=f(t, s, x(s)) & +\left(\frac{k}{n}-x(s)\right) \frac{\partial}{\partial x} f(t, s, x(s)) \\
& +\frac{1}{2}\left(\frac{k}{n}-x(s)\right)^{2} \frac{\partial^{2}}{\partial x^{2}} f(t, s, x(s))+\left(\frac{k}{n}-x(s)\right)^{2} h\left(t, s, \frac{k}{n}-x(s)\right) .
\end{aligned}
$$

Thus, we can write

$$
\begin{aligned}
n^{\alpha}\left|\left(N U S_{n} F\right)(x)-F(x)\right|= & n^{\alpha}\left|\int_{0}^{1} \sum_{k=0}^{n}\left\{H_{n}\left(f\left(t, s, \frac{k}{n}\right) d s\right)-f(t, s, x(s)) d s\right\} \varphi_{k, n}(x(s)) d s\right| \\
\leq & n^{\alpha} \sum_{k=0}^{n} \psi\left(\left|\int_{0}^{1} f\left(t, s, \frac{k}{n}\right) d s-\int_{0}^{1} f(t, s, x(s)) d s\right|\right) \varphi_{k, n}(x(s)) \\
& +n^{\alpha}\left|\sum_{k=0}^{n}\left\{H_{n}\left(\int_{0}^{1} f(t, s, x(s)) d s\right)-\int_{0}^{1} f(t, s, x(s)) d s\right\} \varphi_{k, n}(x(s))\right| \\
= & : I_{1}(x)+I_{2}(x) .
\end{aligned}
$$

By assumption (P.2), $I_{2}(x)$ tends to zero. We can estimate the first term in the following way. Using sub-additivity of the function $\psi(x), x \geq 0$, we have

$$
\begin{aligned}
I_{1}(x)= & n^{\alpha} \sum_{k=0}^{n} \psi\left(\mid\left(\frac{k}{n}-x(s)\right) \frac{\partial}{\partial x} f(t, s, x(s))+\frac{1}{2}\left(\frac{k}{n}-x(s)\right)^{2} \frac{\partial^{2}}{\partial x^{2}} f(t, s, x(s))\right. \\
& \left.+\left(\frac{k}{n}-x(s)\right)^{2} h\left(t, s, \frac{k}{n}-x(s)\right) \mid\right) \varphi_{k, n}(x(s)) \\
\leq & n^{\alpha} \sum_{k=0}^{n} \psi\left(\left|\frac{k}{n}-x(s)\right|\left|\frac{\partial}{\partial x} f(t, s, x(s))\right|\right) \varphi_{k, n}(x(s)) \\
& +n^{\alpha} \sum_{k=0}^{n} \psi\left(\frac{1}{2}\left(\frac{k}{n}-x(s)\right)^{2}\left|\frac{\partial^{2}}{\partial x^{2}} f(t, s, x(s))\right|\right) \varphi_{k, n}(x(s)) \\
& +n^{\alpha} \sum_{k=0}^{n} \psi\left(\left(\frac{k}{n}-x(s)\right)^{2}\left|h\left(t, s, \frac{k}{n}-x(s)\right)\right|\right) \varphi_{k, n}(x(s))
\end{aligned}
$$




$$
\begin{aligned}
\leq & \psi\left(\left|\frac{\partial}{\partial x} f(t, s, x(s))\right|\right) n^{\alpha} \sum_{k=0}^{n} \psi\left(\left|\frac{k}{n}-x(s)\right|\right) \varphi_{k, n}(x(s)) \\
& +\psi\left(\frac{1}{2}\left|\frac{\partial^{2}}{\partial x^{2}} f(t, s, x(s))\right|\right) n^{\alpha} \sum_{k=0}^{n} \psi\left(\left(\frac{k}{n}-x(s)\right)^{2}\right) \varphi_{k, n}(x(s)) \\
& +n^{\alpha} \sum_{k=0}^{n} \psi\left(\left(\frac{k}{n}-x(s)\right)^{2}\left|h\left(t, s, \frac{k}{n}-x(s)\right)\right|\right) \varphi_{k, n}(x(s)) \\
\leq & \psi\left(\left|\frac{\partial}{\partial x} f(t, s, x(s))\right|\right) n^{\alpha} \psi\left(\sum_{k=0}^{n}\left|\left(\frac{k}{n}-x(s)\right)\right| \varphi_{k, n}(x(s))\right) \\
& +\psi\left(\left|\frac{\partial^{2}}{\partial x^{2}} f(t, s, x(s))\right|\right) n^{\alpha} \psi\left(\sum_{k=0}^{n}\left(\frac{k}{n}-x(s)\right)^{2} \varphi_{k, n}(x(s))\right) \\
& +n^{\alpha} \sum_{|k / n-x(s)|<\delta} \psi\left(\left(\frac{k}{n}-x(s)\right)^{2}\left|h\left(t, s, \frac{k}{n}-x(s)\right)\right|\right) \varphi_{k, n}(x(s)) \\
& +n^{\alpha} \sum_{|k / n-x(s)| \geq \delta} \psi\left(\left(\frac{k}{n}-x(s)\right)^{2}\left|h\left(t, s, \frac{k}{n}-x(s)\right)\right|\right) \varphi_{k, n}(x(s)) \\
= & : I_{1,1}(x)+I_{1,2}(x)+I_{1,3}(x)+I_{1,4}(x) .
\end{aligned}
$$

In virtue of Jensen's Inequality, we immediately have

and

$$
\begin{aligned}
I_{1,1}(x) & =\psi\left(\left|\frac{\partial}{\partial x} f(t, s, x(s))\right|\right) n^{\alpha} \psi\left(\sum_{k=0}^{n}\left|\left(\frac{k}{n}-x(s)\right)\right| \varphi_{k, n}(x(s))\right) \\
& =\psi\left(\left|\frac{\partial}{\partial x} f(t, s, x(s))\right|\right) n^{\alpha} \psi\left(M_{1}\left(\varphi_{k, n} x(s)\right)\right),
\end{aligned}
$$

$$
\begin{aligned}
I_{1,2}(x) & =\psi\left(\frac{1}{2}\left|\frac{\partial^{2}}{\partial x^{2}} f(t, s, x(s))\right|\right) n^{\alpha} \psi\left(\sum_{k=0}^{n}\left(\frac{k}{n}-x(s)\right)^{2} \varphi_{k, n}(x(s))\right) \\
& =\psi\left(\frac{1}{2}\left|\frac{\partial^{2}}{\partial x^{2}} f(t, s, x(s))\right|\right) n^{\alpha} \psi\left(M_{2}\left(\varphi_{k, n} x(s)\right)\right) .
\end{aligned}
$$

Now we estimate the terms $I_{1,3}(x)$ and $I_{1,4}(x)$, respectively. Let $\varepsilon>0$ be fixed. There exists a $\delta>0$ such that $\psi(|h(t, s, y)|) \leq \varepsilon$ for every $|y| \leq \delta$. Hence,

$$
I_{1,3}(x)=n^{\alpha} \sum_{|k / n-x(s)|<\delta} \psi\left(\left(\frac{k}{n}-x(s)\right)^{2}\left|h\left(t, s, \frac{k}{n}-x(s)\right)\right|\right) \varphi_{k, n}(x(s)) \leq \varepsilon H(t)
$$

for a sufficiently large $n$. Moreover, choosing a constant $C>0$ such that $\psi(|h(t, s, y)|) \leq C$ for every $|y| \geq \delta$, we have

$$
\begin{aligned}
I_{1,4}(x) & =n^{\alpha} \sum_{|k / n-x(s)| \geq \delta} \psi\left(\left(\frac{k}{n}-x(s)\right)^{2}\left|h\left(t, s, \frac{k}{n}-x(s)\right)\right|\right) \varphi_{k, n}(x(s)) \\
& \leq n^{\alpha} C \psi\left(M_{2}\left(\varphi_{k, n} x(s)\right)\right)=o(1) .
\end{aligned}
$$

In view of the assumption (14), one has

$$
\begin{aligned}
\limsup _{n \rightarrow \infty} n^{\alpha}\left|\left(\operatorname{NUS}_{n} F\right) x(t)-F x(t)\right| & \leq \psi\left(\left|\frac{\partial}{\partial x} f(t, s, x(s))\right|\right) l_{1}(x(s)) \\
& +\psi\left(\frac{1}{2}\left|\frac{\partial^{2}}{\partial x^{2}} f(t, s, x(s))\right|\right) l_{2}(x(s)) .
\end{aligned}
$$

This completes the proof of the theorem. 


\section{References}

[1] Acar T., Costarelli D., Vinti G. Linear prediction, simultaneous approximation by m-th order Kantorovich type sampling series. Banach J. Math. Anal. 2020, 14 (4), 1481-1508. doi:10.1007/s43037-020-00071-0

[2] Angeloni L., Costarelli D., Vinti G. A characterization of the convergence in variation for the generalized sampling series. Ann. Acad. Sci. Fenn. Math. 2018, 43, 755-767. doi:10.5186/aasfm.2018.4343

[3] Angeloni L., Costarelli D., Vinti G. A characterization of the absolute continuity in terms of convergence in variation for the sampling Kantorovich operators. Mediterr. J. Math. 2019, 16 (2), 44. doi:10.1007/s00009-019-1315-0

[4] Angeloni L., Vinti G. Discrete operators of sampling type, approximation in $\varphi$-variation. Math. Nachr. 2018, 291 (4), 546-555. doi:10.1002/mana.201600508

[5] Bardaro C., Mantellini I. Asymptotic expansion of generalized Durrmeyer sampling type series. Jaen J. Approx. 2014, 6 (2), 143-165.

[6] Bardaro C., Mantellini I. A Voronovskaya-type theorem for a general class of discrete operators. Rocky Mountain J. Math. 2009, 39 (5), 1411-1442. doi:10.1216/RMJ-2009-39-5-1411

[7] Bardaro C., Mantellini I. A note on the Voronovskaja theorem for Mellin-Fejer convolution operators. Appl. Math. Lett. 2011, 24 (12), 2064-2067. doi:10.1016/j.aml.2011.05.043

[8] Bardaro C., Faina L., Mantellini I. Quantitative Voronovskaja formulae for generalized Durrmeyer sampling type series. Math. Nachr. 2016, 289 (14-15), 1702-1720. doi:10.1002/mana.201500225

[9] Bardaro C., Karsli H., Vinti G. Nonlinear integral operators with homogeneous kernels: pointwise approximation theorems. Appl. Anal. 2011, 90 (3-4), 463-474. doi:10.1080/00036811.2010.499506

[10] Bardaro C., Musielak J., Vinti G. Nonlinear Integral Operators and Applications. De Gruyter, Berlin, New York, 2003.

[11] Bardaro C., Mantellini I., Stens R., Vautz J., Vinti G. Generalized sampling approximation for multivariate discontinuous signals, application to image processing. In: Zayed A., Schmeisser G. (Eds.) New Perspectives on Approximation and Sampling Theory. Applied and Numerical Harmonic Analysis. Birkhäuser, Cham, 2014, 87-114.

[12] Butzer P.L., Nessel R.J. Fourier Analysis and Approximation, V.1 One-Dimensional Theory. Academic Press, New York, London, 1971.

[13] Butzer P.L., Stens R.L. Sampling theory for not necessarily band-limited functions: an historical overview. SIAM Rev, 1992, 34 (1), 40-53.

[14] Butzer P.L., Ries S., Stens R.L. Approximation of continuous, discontinuous functions by generalized sampling series. J. Approx. Theory 1987, 50 (1), 25-39. doi:10.1016/0021-9045(87)90063-3

[15] Butzer P.L., Stens R.L. Linear prediction by samples from the past. In: Marks R.J. (Ed.) Advanced Topics in Shannon Sampling and Interpolation Theory. Sringer, New York, 1993, 157-183.

[16] Costarelli D., Vinti G. Inverse results of approximation, the saturation order for the sampling Kantorovich series. J. Approx. Theory 2019, 242, 64-82. doi:10.1016/j.jat.2019.03.001

[17] Costarelli D., Vinti G. An inverse result of approximation by sampling Kantorovich series. Proc. Edinb. Math. Soc. 2019, 62 (1), 265-280. doi:10.1017/S0013091518000342

[18] Costarelli D., Minotti A.M., Vinti G. Approximation of discontinuous signals by sampling Kantorovich series. J. Math. Anal. Appl. 2017, 450 (2), 1083-1103. doi:10.1016/j.jmaa.2017.01.066

[19] Costarelli D., Seracini M., Vinti G. A comparison between the sampling Kantorovich algorithm for digital image processing with some interpolation, quasi-interpolation methods. Appl. Math. Comp. 2020, 374, 125046. doi:10.1016/j.amc.2020.125046

[20] Demkiv I.I. On Approximation of the Urysohn operator by Bernstein type operator polynomials. Visn. Lviv. Univ., Ser. Appl. Math. Comp. Sci. 2000, 2, 26-30. 
[21] Karsli H. Some convergence results for nonlinear singular integral operators. Demonstr. Math. 2013, 46 (4), 729 740. doi:10.1515/dema-2013-0487

[22] Karsli H. Convergence and rate of convergence by nonlinear singular integral operators depending on two parameters. Appl. Anal. 2006, 85 (6-7), 781-791. doi:10.1080/00036810600712665

[23] Karsli H. Approximation by Urysohn type Meyer-König and Zeller operators to Urysohn integral operators. Results Math. 2017, 72 (3), 1571-1583. doi:10.1007/s00025-017-0729-x

[24] Karsli H. Approximation results for Urysohn-type nonlinear Bernstein operators. In: Mohiuddine S., Acar T. (Eds.) Advances in Summability and Approximation Theory. Springer, Singapore, 2018, 223-241.

[25] Karsli H. Approximation results for Urysohn type two dimensional nonlinear Bernstein operators. Const. Math. Anal. 2018, 1 (1), 45-57. doi:10.33205/cma.453027

[26] Karsli H. Voronovskaya-type theorems for Urysohn type nonlinear Bernstein operators. Math. Methods Appl. Sci. 2019, 42 (16), 5190-5198. doi:10.1002/mma.5261

[27] Karsli H. Some approximation properties of Urysohn type nonlinear operators. Stud. Univ. Babeş-Bolyai Math. 2019, 64 (2), 183-196.

[28] Karsli H. On Urysohn type generalized sampling operators. Dolomites Res. Notes Approx. 2021,14 (2), $58-67$. doi:10.14658/pupj-drna-2021-2-8

[29] Karsli H., Altin H.E. A Voronovskaya-type theorem for a certain nonlinear Bernstein operators. Stud. Univ. BabeşBolyai Math. 2015, 60 (2), 249-258.

[30] Makarov V.L., Demkiv I.I. Approximation of the Urysohn operator by operator polynomials of Stancu type. Ukrainian Math. J. 2012, 64 (3), 356-386. doi:10.1007/s11253-012-0652-y (translation of Ukrain. Mat. Zh. 2012, 64 (3), 318-343. (in Ukrainian))

[31] Orlova O., Tamberg G. On approximation properties of generalized Kantorovich-type sampling operators. J. Approx. Theory 2016, 201, 73-86. doi:10.1016/j.jat.2015.10.001

[32] Ries S., Stens R.L. Approximation by generalized sampling series. In: Proc. of the Intern. Conf. on Constructive Theory of Functions, Varna, Bulgaria, May 27-June 2, 1984. Publ. House Bulgarian Acad. of Sci., Sofia, 1984, 746-756.

[33] Urysohn P. On one type of nonlinear integral equations. Mat. Sb. 1923, 31 (2), 236-255. (in Russian)

Карслі Х. Асимптотичні властивості узагальнених операторів вибірки типу Урисона // Карпатські матем. публ. - 2021. - Т.13, №3. - С. 631-641.

Ця стаття є продовженням дослідження властивостей збіжності узагальнених операторів вибірки типу Урисона, означених автором у роботі [Dolomites Res. Notes Approx. 2021, 14 (2), 58-67]. Основна увага зосереджена на дослідженні асимптотичних властивостей разом із деякими теоремами типу Вороновської для лінійних та нелінійних аналогів узагальнених операторів вибірки типу Урисона.

Ключові слова і фрази: узагальнений оператор вибірки, лінійний та нелінійний аналоги узагальненого оператора вибірки типу Урисона, асимптотичне розвинення, теорема типу Вороновської. 